\title{
Beyond Viral Interferon Regulatory Factors: Immune Evasion Strategies
}

\author{
Jinjong Myoung ${ }^{1}$, Shin-Ae Lee ${ }^{2}$, and Hye-Ra Lee ${ }^{3 *}$ \\ ${ }^{1}$ Korea Zoonosis Research Institute, Genetic Engineering Research Institute and Department of Bioactive Material Science, College of Natural \\ Science, Jeonbuk National University, Jeonju 54531, Republic of Korea \\ ${ }^{2}$ Department of Molecular Microbiology and Immunology, Keck School of Medicine, University of Southern California, Los Angeles, \\ California, USA \\ ${ }^{3}$ Department of Biotechnology and Bioinformatics, College of Science and Technology, Korea University, Sejong 30019, Republic of Korea
}

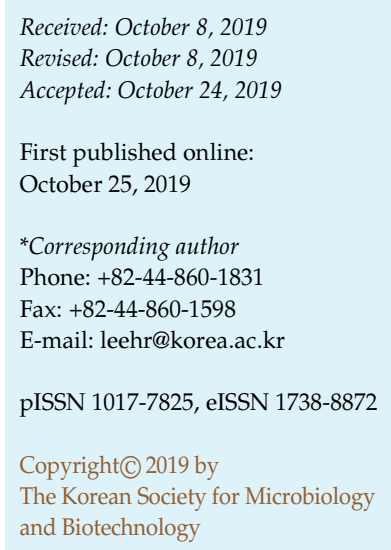

The innate immune response serves as a first-line-of-defense mechanism for a host against viral infection. Viruses must therefore subvert this anti-viral response in order to establish an efficient life cycle. In line with this fact, Kaposi's sarcoma-associated herpesvirus (KSHV) encodes numerous genes that function as immunomodulatory proteins to antagonize the host immune system. One such mechanism through which KSHV evades the host immunity is by encoding a viral homolog of cellular interferon (IFN) regulatory factors (IRFs), known as vIRFs. Herein, we summarize recent advances in the study of the immunomodulatory strategies of KSHV vIRFs and their effects on KSHV-associated pathogenesis.

Keywords: KSHV, viral interferon regulatory factor, immune evasion strategy, PRR-mediated signaling pathway, apoptosis pathway

\section{Introduction}

Kaposi's sarcoma-associated herpesvirus (KSHV) is a DNA tumor virus and identified as the causative agent of Kaposi's sarcoma (KS), primary effusion lymphoma (PEL), and multicentric Castleman's disease (MCD) [1-3]. In order to efficiently establish life-long persistent infection as well as KSHV-associated pathogenesis, KSHV encodes numerous immunomodulatory proteins, within a large portion of its genome, that overcome the host immune system to ensure viral persistence [4]. As part of its strategy, KSHV harbors viral interferon (IFN) regulatory factors (vIRFs) that have sequence homology with cellular IRFs.

Cellular IRFs are well-known transcriptional factors that bind and activate IFN-responsible promoters. Activated IFN response upon virus infection subsequently leads to phosphorylation, dimerization, and nuclear translocation of IRF3/IRF7, which are key factors of type I IFN creating an anti-viral state against viral infection. All cellular IRFs have a conserved N-terminal DNA binding domain (DBD) that contains five tryptophan repeats and a characteristic C-terminal IRF-association domain (IAD) responsible for IRF homo- or heterodimerization, as well as binding with various transcription factors. In addition to their imperative role in the innate immune response, IRFs are also implicated in various physiological processes, such as cellular differentiation, cell growth, and apoptosis. Thus, deregulation of IRFs occurs in numerous pathological conditions, such as viral infection, tumorigenesis, and so on.

To counteract these cellular IRFs, vIRFs have been shown to elicit two main biological consequences: inhibition of the IFN-mediated innate immunity and suppression of the p53mediated apoptosis. Furthermore, more recent mounting data suggested that vIRFs might function as a viral transcriptional factor, although they do not comprise the five conserved tryptophan residues within their predicted DBD region, unlike cellular IRFs. Thus, the aim of this review is to comprehensively understand the possible modes of action of vIRFs in KSHV-associated pathogenesis as well as their life cycles.

\section{Immune Signaling Pathway}

The innate immune response is the first line of host defense mechanism against viral infections. Therefore, it is essential for the virus to overcome the innate immune 
response in order to establish a successful viral life cycle within the host. Upon virus invasion, host pattern recognition receptors (PRRs) immediately sense pathogenassociated molecular patterns (PAMPs), which are specific molecular features associated with pathogens. Currently, several PRRs have been identified, including cytosolic sensors, toll-like receptors (TLRs), and nucleotide-binding oligomerization domain (NOD)-like receptors (NLRs). These PRRs comply with the general mechanism of signaling: they recognize PAMPs, and immediately trigger an intracellular signal transduction pathway that activates the gene expression and synthesis of inflammatory cytokines, including IFNs. Subsequently, secreted IFNs bind to the IFN receptors, further activating the janus kinase-signal transducer and activator of transcription (JAK-STAT) pathway, leading to expression of IFN-stimulated genes (ISGs). Ultimately, these signaling events amplify the innate immune signaling and coordinate adaptive immune response within the cell to build up an anti-viral state. Therefore, in order for the virus to efficiently complete its life cycle in the host, KSHV evolutionally employs numerous strategies to evade the host immune signaling pathways.

\section{vIRF1 (K9)}

KSHV open reading frame (ORF) K9 encodes vIRF1, which effectively downregulates the transcriptional activity of IFN promoter, ISG promoter, and gamma interferonactivated sequence (GAS)-element [5]. These inhibitory effects of vIRF1 on IFN-mediated signaling occur via interaction of vIRF1 with cellular proteins. vIRF1 binds p300 and selectively prevents p300/CBP-IRF3 complex to suppress the IRF3-mediated transcriptional activity [6, 7]. Interestingly, although vIRF1 binds to IRF3, previous studies have shown that vIRF1-IRF3 interaction did not alter IRF3-mediated homo- or heterodimerization, nuclear translocation, nor DNA binding activity. However, it has recently been reported by Jacobs et al. that VIRF1 reduced phosphorylation of IRF3 and nuclear localization of IRF3 upon TLR3-mediated upregulation of IFN- $\beta$ expression [8]. In addition, the region between amino acids 224 and 349 of vIRF1 was responsible for interaction with cellular interferon-stimulated gene 15 (ISG15) E3 ligase, HERC5, leading to decreased ISGylation of target cellular proteins [5]. Notably, knockdown of ISG15 induced KSHV reactivation to its lytic cycle [5]. As is the case with most DNA viruses, cGMP-AMP synthase (cGAS) can recognize viral DNA in the cytoplasm and generate cGMP-AMP (cGAMP) that activates STING to cause production of type I IFN [9]. KSHV, thus, utilizes vIRF1 to ablate the cGAS-
STING-mediated IFN production via inhibiting phosphorylation of STING [10]. Interestingly, knockdown of vIRF1 with siRNA against vIRF1 in KSHV-infected cells shows suppression of viral lytic replication during reactivation conditions [10]. Collectively, these indicate that vIRF1 utilized several different strategies to efficiently perturb the anti-IFN activities for establishment of primary infection as well as replication.

\section{vIRF2 (K11 and K11.1)}

vIRF2 encoded by K11 and K11.1 has different isoforms as a result of alternative splicing of exons K11 and K11.1. These isoforms of vIRF2 localize both in the nucleus and the cytoplasm [11]. Interestingly, vIRF2 suppresses the transcriptional activity of IFN-stimulated promoter and IFN- $\beta$ promoter by interaction with both IRF1 and IRF3, but not IRF7 [12, 13]. Furthermore, vIRF2 interacts with IRF2, ICSBP, RelA (p65), and p300, leading to inhibition of IFN- $\alpha$ gene expression as well as early pro-inflammatory cytokine expression $[12,13]$. In order to efficiently escape the type I IFN-driven antiviral response, vIRF2 recruits caspase-3 to IRF3 and induces IRF3 degradation [14]. In addition, the short form of vIRF2 inhibits late-state IFN response via preventing protein kinase-R (PKR) activation, which is induced by binding of dsRNA created during viral replication [15]. Activated PKR leads to phosphorylation of the eukaryotic translation initiation factor $\alpha(\mathrm{eIF} 2 \alpha)$ and subsequently inhibits cellular mRNA synthesis, preventing viral protein synthesis. Thereby, KSHV vIRF2 comprehensively suppressed early and late anti-viral states.

\section{vIRF3 (K10.5)}

Unlike vIRF1 and vIRF2, vIRF3 facilitates activation of both IRF3- and IRF7-mediated type I IFN via interaction with IRF3, IRF7, and p300 [16]. Uniquely, vIRF3 is involved in enhanceosome activity, leading to increased interaction of IRF3 and IRF7 to the IFN- $\alpha$ promoter in infected cells [16]. Surprisingly, the same group also reported that vIRF3 acts as a dominant negative factor of both IRF3 and IRF7 to suppress virus-induced type I IFN promoter activity [17]. Later, Joo et al. also showed that vIRF3 suppressed IRF7mediated IFN- $\alpha$ production by binding specifically with the DNA-binding domain (DBD) of IRF7 in 293T transfected cells upon Sendai virus treatment [18]. vIRF3 also interacts with IRF5, a member of the IRF family that was first identified as a transcriptional factor of type I IFN. vIRF3 interaction with IRF5 inhibits IRF5 from binding to the ISRE region of the promoter, and leading to suppression of IRF5-mediated IFN-response gene activation [19]. 


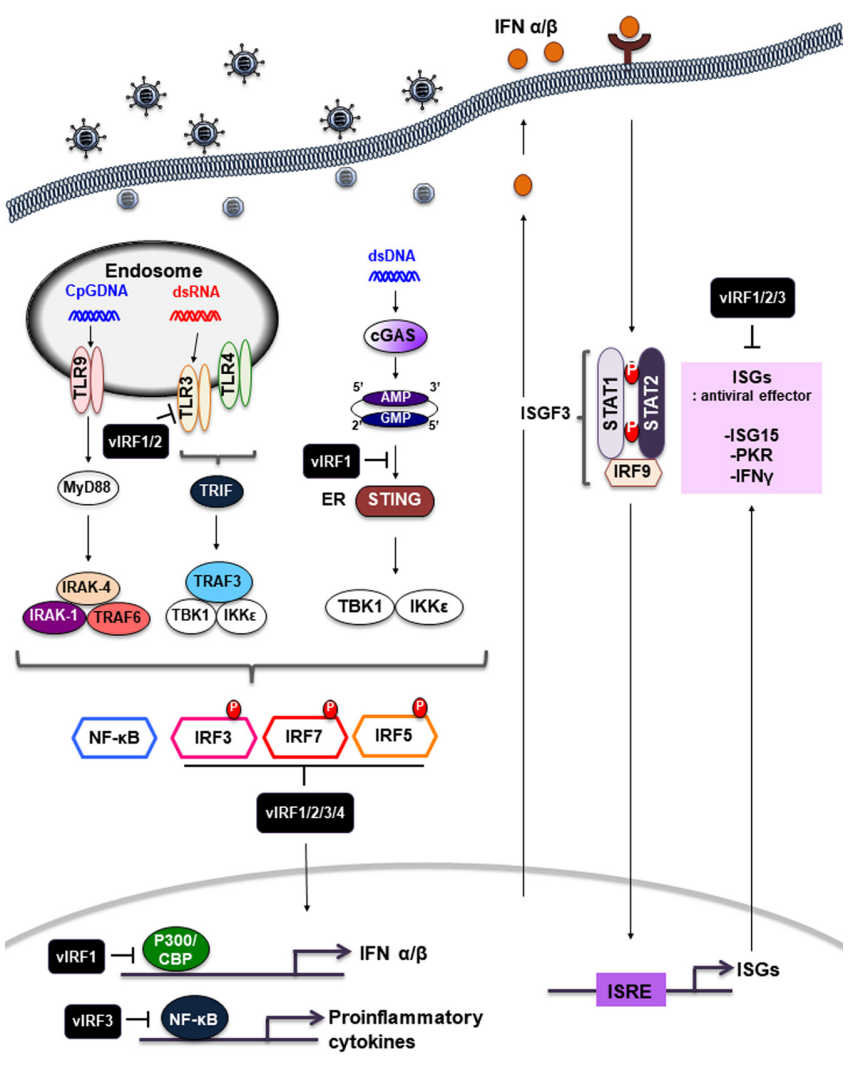

Fig. 1. KSHV vIRFs deregulate PRR-mediated innate immune responses.

Upon KSHV infection into the host cells, pattern recognition receptors (PRRs) recognize the pathogen-associated molecular patterns (PAMPs) of the pathogen and subsequently turn on signaling by cellular IRFs, including IRF3, IRF5, IRF7, and numerous IFN-stimulated genes (ISGs) including PKR, ISG15, and IFN- $\gamma$. KSHV vIRFs utilize various different strategies to combat the highly regulated PRR-mediated immune responses by the host. Black square boxes represented four kinds of vIRFs encoded KSHV.

\section{vIRF4 (K10)}

A recent study demonstrated for the first time that vIRF4 has potential to affect the IFN-mediated innate immunity. vIRF4, unlike vIRF3, specifically interacts with IRF7, but not IRF3. Binding of vIRF4 to IRF7 prevents IRF7 dimerization and suppresses the IFN- $\alpha$-associated IFN signaling pathway that escapes the host antiviral activity [20].

\section{Apoptosis Signaling Pathway}

\section{Caspase-Mediated Apoptosis}

Apoptosis, the well-known programmed cell death, can be initiated either by an intrinsic pathway or an extrinsic pathway. During stress conditions, such as viral infection, these pathways subsequently activate initiator caspases and executioner caspases that ultimately induce cell death. The extrinsic pathway is initiated by binding of ligands to death receptors (DR), such as tumor necrosis factor (TNF) $\alpha$ receptor (TNF $\alpha \mathrm{R})$, Fas receptor, and so on. Upon binding of the ligand, the receptors undergo oligomerization and subsequently recruit adaptor proteins that activate the caspase cascades [21]. For instance, interaction of Fas ligand (FasL) with Fas receptor induces trimerization of Fas receptor, leading to binding of the Fas-associated DD (FADD) adaptor protein that can interact with initiator caspase-8 through the death effector domains [21]. Caspase- 8 then undergoes autoproteolysis to generate activated caspase-8, which consequently initiates extrinsic apoptosis by direct cleavage of effector caspases-3, -6, and -7. Moreover, activated caspase- 8 can cleave Bid, one of the pro-apoptotic Bcl-2 family members, ultimately leading to release of cytochrome $C$ from the mitochondria [22]. Release of cytochrome $\mathrm{C}$ can also activate caspase- 3 through the formation of an apoptosome complex containing Apaf-1/ cytochrome C/caspase-9 [21, 22]. Consequently, these active executioner caspases cleave vital cellular substrates that lead to cell death. In order to combat the tightly regulated extrinsic and intrinsic apoptosis pathways, KSHV has employed various strategies to deregulate expression of CD95 ligand (CD95L, also known as FasL) and proapoptotic activities via its vIRF1 and vIRF2.

vIRFs As noted earlier, both vIRF1 and vIRF2 primarily behave as negative regulators of the extrinsic and intrinsic apoptosis pathways. Firstly, vIRF1 suppresses the activation-induced cell death by targeting CD95L. vIRF1 not only inhibits IRF1-mediated induction of CD95L through binding to the regulatory IRF-1-dependent domains (PRIDD), but also downregulates $\mathrm{T}$ cell receptor (TCR)-mediated CD95L expression $[23,24]$. Consistent with vIRF1, vIRF2 also inhibits TCR-mediated CD95L induction, although its underlying molecular mechanism is not clear [23]. CD95L is predominantly expressed in activated $\mathrm{T}$ cell and is induced upon stimulation of either IFN- $\gamma$ or T cell receptor (TCR) $[25,26]$. Upon binding of CD95L to its receptor CD95, an assemblage of the death-inducing signaling complex (DISC) occurs, activating caspase- 8 to initiate the execution of apoptosis $[25,26]$. Very recently, Kim et al. showed that vIRF2 inhibits the IRF3-mediated caspase-3 activity [27]. Uniquely, vIRF1, among other vIRFs, has been reported to be the only one to suppress mitochondrial-mediated apoptosis signaling. vIRF1 inhibits the mitochondriaassociated intrinsic apoptosis pathway by interacting with Bcl-2 homology 3 (BH3) domain of Bid and Bim, which 
have pro-apoptotic activity, resulting in suppression of cellular apoptotic signals [28]. The pro-apoptotic molecules Bid and Bim cause permeabilization of the outer mitochondrial membrane, leading to efflux of cytochrome C, which binds the adaptor Apaf- 1 and the initiator caspase- 9 in the cytosol to form the apoptosome complex. Subsequently, activation of caspase- 3 promotes the execution of cell death [26]. Interestingly, vIRF1 specifically sequesters Bim from the mitochondria to the nucleus, which consequently inhibits the pro-apoptotic function of Bim. However, direct interaction of vIRF1-Bid led to inhibition of Bid-associated mitochondrial permeabilization. Consistently, depletion of both Bid and Bim in KSHV-infected cells showed increase in KHSV replication, confirming the anti-regulatory role of Bid and Bim in KSHV proliferation [28]. The Bim-binding domain (BBD) of vIRF1 also interacts with other BH-3 domain-containing proteins such as Bik, Bmf, and Noxa [28]. Another anti-apoptotic mechanism by which vIRF1 acts is via its interaction with the membrane lipid, cardiolin, targeting the mitochondrial detergent-resistant membrane fraction to hinder the MAVS-mediated apoptosis and antiviral responses [29].

\section{p53-Mediated Apoptosis}

The tumor suppressor gene p53 has widely been accepted as a crucial regulatory factor in one of the major apoptosis signaling pathways. p53 serves as a transcription factor that regulates the expression of numerous genes, including those involved in apoptosis or cell cycle arrest upon various cellular stress conditions, such as viral infection [30]. Therefore, it is not surprising that host cells upregulate and activate p53 upon virus infection. In order for the viruses to establish a successful life cycle within the host cells, viruses, in turn, hijack the p53-mediated cell death and cause irreversible cell cycle arrest [31].

Due to the crucial role of p53 in regulating various cellular processes, the gene is kept under tight regulation [32, 33]. In normal physiological conditions, p53 is maintained at low steady state levels by murine double minute 2 (MDM2)-mediated ubiquitin degradation. In addition, MDM2 has also been shown to mask the transactivation domain of p53 to inhibit its transcriptional abilities [32, 33]. On the other hand, activated p53 transcribes the gene expression of its negative regulator, $M D M 2$, exhibiting an autoregulatory negative feedback loop [32, 33]. Moreover, active p53 is subject to a series of post-translational modifications, including acetylation and phosphorylation. For example, upon DNA damage response, p53 is acetylated by the histone acetylase transferase CREB-binding protein

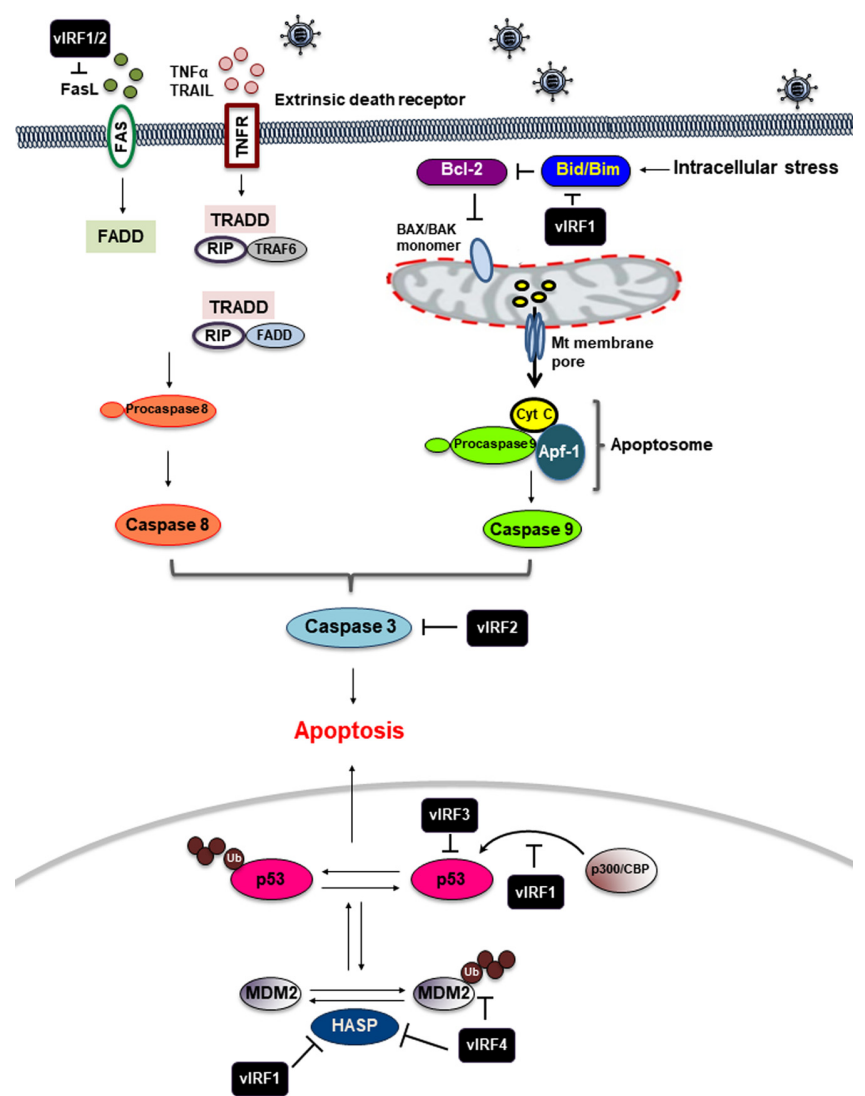

Fig. 2. KSHV vIRFs inhibit intrinsic and extrinsic apoptotic pathways.

Virus infection leads to activation of both extrinsic and intrinsic apoptotic pathways, including p53-mediated apoptotic activities. Hence, KSHV employed vIRFs to counteract and suppress the apoptotic signaling pathways in three distinct ways. Firstly, vIRFs perturb the FasL-mediated extrinsic apoptosis. Secondly, vIRFs inhibit the Bim/Bid-associated intrinsic apoptosis, and lastly, vIRFs downregulate p53-mediated transcriptional activity and protein stability. Black square boxes indicate vIRFs.

(CBP)/p300 and phosphorylated by ataxia telangiectasiamutated (ATM) kinase that correspondingly activate p53 while also stabilizing its protein levels [30].

vIRF1 (K9) vIRF1 employs two different mechanisms to restrain the activities of p53: first, vIRF1 suppresses phosphorylation of p53 on serine 15 residue by blocking the ATM kinase activity via its interaction and subsequently increasing p53 ubiquitination by MDM2, resulting in p53 degradation [34]; second, vIRF1 binds p53 and inhibits p53 acetylation, which consequently inhibits p53-mediated apoptosis due to suppression of p53 gene expression [35]. Later, Chavoshi et al. reported that vIRF1 also interacts with ubiquitin-specific protease 7 (USP7, also known as 
HAUSP) and deregulates its enzymatic activities, leading to inhibition of p53-mediated antiviral activities [36].

vIRF3 (K10.5) vIRF3 also interacts with p53 and downregulates its transcriptional activity, leading to inhibition of p53-mediated apoptosis in B cells. Although vIRF3 interacted with amino acids 290 to 393 of p53 sufficiently under an overexpression system, vIRF3 did not interact with endogenous p53. Therefore, the molecular mechanism underlying how vIRF3 coordinates p53 activity still remains unclear.

vIRF4 (K10). vIRF4 features a strong anti-apoptotic function by tightly modulating the p53-associated ubiquitination and deubiquitination system to effectively control the p53 protein levels. The C-terminal region of vIRF4 (amino acids 606 to 758) interacts with MDM2 E3 ubiquitin ligase. This interaction stabilizes MDM2, ultimately leading to decreased p53 protein levels, due to enhancement of p53 ubiquitination. Ultimately, vIRF4 inhibits p53-mediated apoptosis to create a favorable environment for the virus to replicate within the host [37]. The protein level of p53 has been well characterized as very important in the host defense signaling against viral infection. Additionally, HAUSP gained attention as a crucial component that regulates p53 protein levels through dual control of p53 and its negative regulator, MDM2. Surprisingly, vIRF4 also evolutionally employed targeting HAUSP to suppress the p53-mediated tumor suppressor activities. This time, the $\mathrm{N}$-terminal region of vIRF4 was responsible for binding both the TRAF domain and catalytic domain of HAUSP, leading to inhibition of substrate binding as well as deubiquitinase activity of HAUSP [38]. Furthermore, although vIRF4 interacts with both MDM2 and HAUSP to control p53 protein levels, data indicated that vIRF4-MDM2 interaction was more superior in downregulating p53 than vIRF4-HAUSP [38]. Taken together, the anti-apoptotic mechanism of vIRF4 summarizes as the N-terminal arm of vIRF4 grabbing and holding HAUSP, while the C-terminal arm of vIRF4 targets and inhibits MDM2. More importantly, these two activities are genetically separable but functionally addictive, suggesting vIRF4 as a crucial virulence factor in anti-apoptotic function.

\section{Potential Viral Transcriptional Factor}

Like other herpesviruses, KSHV displays two different phases of viral life cycle: latent and lytic [31]. In particular, the latent life cycle displays persistency due to maintaining its genome as a circular episome in the nucleus whereby only a few genes get transcribed from the latency transcript cluster [39]. Remarkably, this latent phase has reversibility properties as well as a capability to inhibit the lytic cycle. Hence, under appropriate circumstances, the lytic phase can be reactivated, expressing three different kinetic classes of genes, the immediate early (IE), early (E), and late (L) transcripts, resulting in production of infectious progeny viruses [39].

As described above, we have discussed how vIRFs function as immunomodulatory factors in two distinct mechanisms, both of which reshape cellular circumstances favorably for the virus to establish efficient initial infection. vIRFs have significant homology with cellular IRFs, which are a family of transcriptional factors that play roles as transcription activators or repressors. All the IRFs encode an N-terminal DNA-binding domain (DBD), which consists of five tryptophan repeats, and a C-terminal IRFassociation domain (IAD) that engages in interactions with different transcriptional factors, co-factors, and other IRFs [40] (Fig. 3). Unlike cellular IRFs, KSHV vIRFs lack one or two tryptophan residues [41] (Fig. 3). However, evidence has suggested that vIRFs have the capability of directly or indirectly binding to the promoter regions and functioning as authentic transcriptional factors themselves. Hence, it is important to describe the potential DNA-binding activity of vIRFs as shown with their cellular counterparts, which may enlighten the novel roles of vIRFs as viral transcription factors in modulating both host and viral gene expression to benefit viral life cycle or contribute to KSHV-associated pathogenesis.

\section{vIRF1 (K9)}

It has been reported that vIRF1 can interact with DNA oligonucleotide via a consensus sequence of DNA binding motif, which eventually leads to binding to the promoter region of $\mathrm{K} 3$ in the KSHV genome [42]. Recent crystal structure and thermal stability shift assay (TSSA) also revealed that vIRF1 DBD, but not full-length vIRF1, can bind to the phosphate backbone of DNA [43]. In addition, vIRF1 negatively regulates p300/CBP and Smads, which are known transcriptional activators, by directly interacting with them [44]. These studies demonstrate that vIRF1 functions as a transcriptional regulator by either forming a vIRF1-DNA or a vIRF1-protein complex to differentially modulate viral as well as cellular gene expression.

\section{vIRF2 (K11 and K11.1)}

In addition to vIRF1, genome-wide chromatin immunoprecipitation coupled with high-throughput sequencing (ChIP-Seq) analysis revealed that vIRF2 is able to bind the 
A
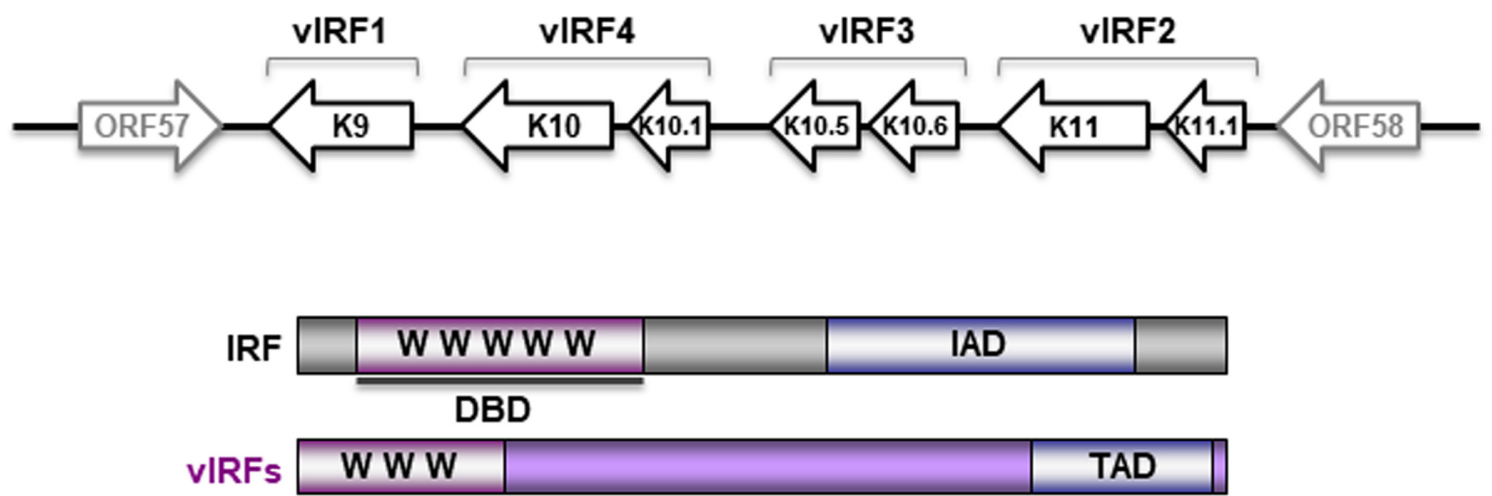

B

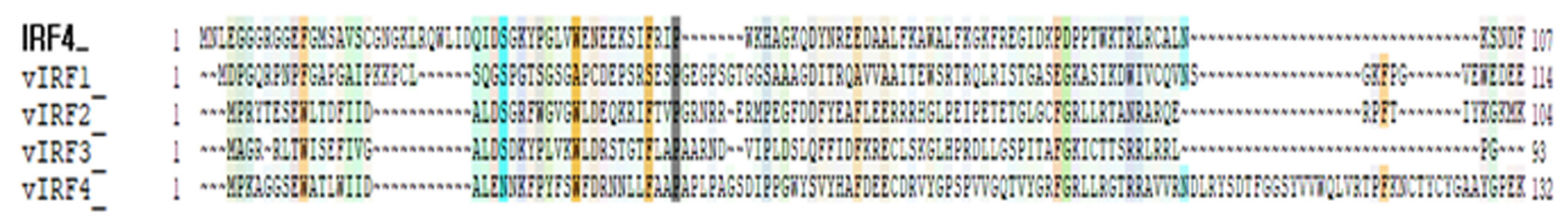

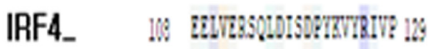

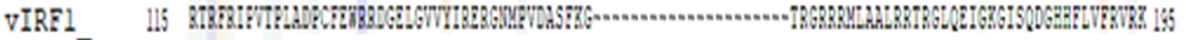

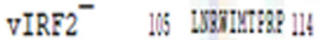

VIRE3 ${ }^{-}$\& $\operatorname{sing}$ ?

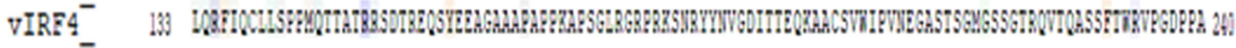

\section{Identity Scores (\%)}

\begin{tabular}{|l|c|c|c|c|c|}
\hline & IRF4_ & vIRF1_ & vIRF2_ & vIRF3_ & vIRF4_ \\
\hline IRF4_ & 100 & 15 & 12 & 12 & 8 \\
\hline URF1_ & 15 & 100 & 10 & 7 & 8 \\
\hline vRF2_- & 12 & 10 & 100 & 24 & 30 \\
\hline vRF3_- & 12 & 7 & 24 & 100 & 18 \\
\hline vRF4_ & 8 & 8 & 30 & 18 & 100 \\
\hline
\end{tabular}

Fig. 3. A brief of the structural organization of vIRFs.

(A) VIRF genes are located between ORF53 and ORF58 of the KSHV genome. Cellular IRFs comprise two major domains, an N-terminal DNA binding domain (DBD) which contains five tryptophan (W) residues, and a C-terminal IRF associated domain (IAD) that can include a transactivation domain (TAD) of IRFs. Similar to cellular IRFs, vIRFs harbor three tryptophan residues at N-terminal region, which function as a putative DNA binding domain, while containing TAD at the C-terminus. (B) Multiple alignment of the DNA binding domains of cellular IRF4 and vIRFs by using CLUSTAL X software.

promoter regions of numerous cellular genes [45]. Notably, VIRF2 DBD (amino acid 7 to 144) was shown to bind to the promoter regions of PIK3C3, HMGCR, HMGCL and subsequently deregulated their expression levels [45]. Importantly, the consequence of such binding contributed to enhancement of KSHV infection and KSHV-associated pathogenesis. Recently, vIRF2 has been implicated in the regulation of early lytic gene expression in KSHV-infected endothelial cells [46]. Depletion of vIRF2 level by siRNA in endothelial cells resulted in inhibition of KSHV lytic gene expression. Using several different KSHV mutants which harbor a stop codon in different sites of VIRF2, it was found that the N-terminal region of vIRF2 containing DBD, was required for suppression of KSHV early lytic gene expression [46]. These data suggested that vIRF2-mediated increase in IFN-response gene transcription may play a crucial role in KSHV life cycle.

\section{vIRF3 (K10.5)}

Recently, vIRF3 has been shown to orchestrate global gene 
expression in lymphatic endothelial cells (LEC) through the interaction with histone deacetylase transferase 5 (HDAC5), which in turn induces hypersprouting formation as well as irregular growth [47]. vIRF3-HDAC5 interaction decreased the phosphorylation of HDAC5, sustaining HDAC5 activity in the nucleus, which ultimately contributed to virusinduced lymphangiogenesis in a LEC-specific manner [47]. Furthermore, remarkably, the expression of vIRF3 induced KS-like spindle morphology in LEC and is easily detected in different KS lesions [47]. These data suggested that alteration of the global gene expression of LEC via vIRF3HDAC5 interaction plays a crucial role in KSHV-associated pathogenesis, like KS. More importantly, this study, unlike previous reports, was the first to show that vIRF3 is a bona fide latent protein that can be detected in both PEL and KS [48].

\section{vIRF4 (K10)}

Studies on vIRF4 modulation of host gene expression via genome-wide analysis have shown that expression of vIRF4 globally affected expression of around 300 cellular genes in BCBL1 cells, which are latently KSHV-infected B cells [49]. Among them, vIRF4 dramatically suppressed cellular IRF4 (cIRF4)-mediated c-Myc gene expression, resulting in contribution of efficient KSHV lytic replication. Chromatin immunoprecipitation together with real-time quantitative PCR analysis assay suggested how vIRF4 deregulates cIRF4 in two independent manners: vIRF4 competes with cIRF4 for the binding region on the $c-M y c$ promoter, and vIRF4 suppresses cIRF4 gene expression with an unknown molecular mechanism [50]. Very recently, $\mathrm{Yu}$ et al. elucidated that vIRF4 also binds to a specific region on cIRF4, leading to a robust downregulation of cIRF4 gene expression [51]. In addition to suppression of cMyc expression, vIRF4-mediated deregulation of cIRF4 enhances B-cell lymphoma 6 (BCL6) expression that greatly activates its transcriptional activity, contributing to KSHV lytic cycle [51]. Indeed, IRF4 is known as a lymphocytespecific IRF family member involved in either positive or negative regulation of its target genes. In line with this, accumulated data indicated that IRF4 is a negative regulator of BCL6 gene expression [52]. vIRF4 DBD also affects the $\beta$-catenin/CBP transcriptionally active complex by inhibiting its accessibility on the cyclin D1 promoter, blocking the G1-S transition [53]. Propidium iodide (PI) staining and bromodeoxyuridine (BrdU) incorporation cell proliferation assay indicated that expression of vIRF4 decreases G1 phase and increases the KSHV DNA copy number [53]. Interestingly, vIRF4 also stimulates activity of the lytic switch protein, RTA, to facilitate KSHV reactivation, contributing as a positive cofactor of RTA [49]. Together, these data show that vIRF4 can generate both a proteinDNA complex and a protein-protein complex, demonstrating its capability to function as a viral transcriptional factor to coordinate both cellular and viral gene expressions.

\section{Conclusion}

We have highlighted the critical roles of vIRFs in blocking the host innate immune response and the apoptosis pathway for efficient establishment of the KSHV life cycle and pathogenesis (Figs. 1 and 2). Most remarkably, we expanded the knowledge of vIRFs as viral transcriptional factors that regulate various signaling pathways via deregulation of different target gene expression either by competing for occupancy of transcriptional factors or sequesteration of transcriptional co-factors on the promoter region. The present knowledge also clearly suggested that vIRFs have ample impacts on both the KSHV and host gene expression, which conjointly contribute to KSHV life cycle as well as KSHV-associated pathogenesis. In fact, rhesus macaque rhadinovirus (RRV), which is closely related to the $\gamma$-herpesvirus KSHV and also encodes eight vIRFs, highlighted the importance of vIRFs in viral pathogenesis, as infection of vIRFs-knockout (KO) RRV in rhesus macaques showed reduced viral loads and significantly decreased B-cell hyperplasia [54, 55]. Thus, future studies on the vIRFs will be necessary to confirm their roles during the viral life cycle and pathogenesis in a genetic context similar with RRV.

\section{Acknowledgements}

Hye-Ra Lee is supported by the grant agency of the NRF2019R1A2C1008512, NRF-2018M3A9H4089656, NRF2016R1D1A1B03931761. We also apologize to the authors whose works were not mentioned due to space limitation.

\section{Conflict of Interest}

The authors have no financial conflicts of interest to declare.

\section{References}

1. Soulier J, Grollet L, Oksenhendler E, Cacoub P, CazalsHatem D, Babinet P, et al. 1995. Kaposi's sarcoma-associated 
herpesvirus-like DNA sequences in multicentric Castleman's disease. Blood 86: 1276-1280.

2. Nador RG, Cesarman E, Chadburn A, Dawson DB, Ansari MQ, Sald J, et al. 1996. Primary effusion lymphoma: a distinct clinicopathologic entity associated with the Kaposi's sarcoma-associated herpes virus. Blood 88: 645-656.

3. Ganem D. 2010. KSHV and the pathogenesis of Kaposi sarcoma: listening to human biology and medicine. J. Clin. Invest. 120: 939-949.

4. Lee HR, Amatya R, Jung JU. 2015. Multi-step regulation of innate immune signaling by Kaposi's sarcoma-associated herpesvirus. Virus Res. 209: 39-44.

5. Li M, Lee H, Guo J, Neipel F, Fleckenstein B, Ozato K, et al. 1998. Kaposi's sarcoma-associated herpesvirus viral interferon regulatory factor. J. Virol. 72: 5433-40.

6. Burysek L, Yeow WS, Lubyova B, Kellum M, Schafer SL, Huang $Y Q$, et al. 1999. Functional analysis of human herpesvirus 8-encoded viral interferon regulatory factor 1 and its association with cellular interferon regulatory factors and p300. J. Virol. 73: 7334-42.

7. Li M, Damania B, Alvarez X, Ogryzko V, Ozato K, Jung JU. 2000. Inhibition of p300 histone acetyltransferase by viral interferon regulatory factor. Mol. Cell Biol. 20: 8254-8263.

8. Jacobs SR, Gregory SM, West JA, Wollish AC, Bennett CL, Blackbourn DJ, et al. 2013. The viral interferon regulatory factors of kaposi's sarcoma-associated herpesvirus differ in their inhibition of interferon activation mediated by toll-like receptor 3. J. Virol. 87: 798-806.

9. West AP, Khoury-Hanold W, Staron M, Tal MC, Pineda CM, Lang SM, et al. 2015. Mitochondrial DNA stress primes the antiviral innate immune response. Nature 520: 553-557.

10. Ma Z, Jacobs SR, West JA, Stopford C, Zhang Z, Davis Z, et al. 2015. Modulation of the cGAS-STING DNA sensing pathway by gammaherpesviruses. Proc. Natl. Acad. Sci. USA 112: E4306-15.

11. Jenner RG, Alba MM, Boshoff C, Kellam P. 2001. Kaposi's sarcoma-associated herpesvirus latent and lytic gene expression as revealed by DNA arrays. J. Virol. 75: 891-902.

12. Fuld S, Cunningham C, Klucher K, Davison AJ, Blackbourn DJ. 2006. Inhibition of interferon signaling by the Kaposi's sarcoma-associated herpesvirus full-length viral interferon regulatory factor 2 protein. J. Virol. 80: 3092-3097.

13. Burysek L, Yeow WS, Pitha PM. 1999. Unique properties of a second human herpesvirus 8-encoded interferon regulatory factor (vIRF-2). J. Hum. Virol. 2: 19-32.

14. Areste C, Mutocheluh M, Blackbourn DJ. 2009. Identification of caspase-mediated decay of interferon regulatory factor-3, exploited by a Kaposi sarcoma-associated herpesvirus immunoregulatory protein. J. Biol. Chem. 284: 23272-23285.

15. Burysek L, Pitha PM. 2001. Latently expressed human herpesvirus 8-encoded interferon regulatory factor 2 inhibits double-stranded RNA-activated protein kinase. J. Virol. 75: 2345-2352.
16. Lubyova B, Kellum MJ, Frisancho AJ, Pitha PM. 2004 Kaposi's sarcoma-associated herpesvirus-encoded vIRF-3 stimulates the transcriptional activity of cellular IRF-3 and IRF-7. J. Biol. Chem. 279: 7643-7654.

17. Lubyova B, Pitha PM. 2000. Characterization of a novel human herpesvirus 8-encoded protein, vIRF-3, that shows homology to viral and cellular interferon regulatory factors. J. Virol. 74: 8194-8201.

18. Joo CH, Shin YC, Gack M, Wu L, Levy D, Jung JU. 2007. Inhibition of interferon regulatory factor 7 (IRF7)-mediated interferon signal transduction by the Kaposi's sarcomaassociated herpesvirus viral IRF homolog vIRF3. J. Virol. 81: 8282-8292.

19. Wies E, Hahn AS, Schmidt K, Viebahn C, Rohland N, Lux A, et al. 2009. The Kaposi's Sarcoma-associated Herpesvirusencoded vIRF-3 inhibits cellular IRF-5. J. Biol. Chem. 284: 8525-8538.

20. Hwang SW, Kim D, Jung JU, Lee HR. 2017. KSHV-encoded viral interferon regulatory factor 4 (vIRF4) interacts with IRF7 and inhibits interferon alpha production. Biochem. Biophys. Res. Commun. 486: 700-705.

21. Green DR, Llambi F. 2005. Cell Death Signaling. Cold Spring Harb Perspect. Biol. 7(12).

22. Willis SN, Adams JM. 2005. Life in the balance: how BH3only proteins induce apoptosis. Curr. Opin. Cell Biol. 17: 617625.

23. Kirchhoff S, Sebens T, Baumann S, Krueger A, Zawatzky R, Li-Weber M, et al. 2002. Viral IFN-regulatory factors inhibit activation-induced cell death via two positive regulatory IFN-regulatory factor 1-dependent domains in the CD95 ligand promoter. J. Immunol. 168: 1226-1234.

24. Peter ME, Hellbardt S, Schwartz-Albiez R, Westendorp MO, Walczak H, Moldenhauer G, et al. 1995. Cell surface sialylation plays a role in modulating sensitivity towards APO-1-mediated apoptotic cell death. Cell Death Differ. 2: 163-171.

25. Peter ME, Hadji A, Murmann AE, Brockway S, Putzbach W, Pattanayak A, et al. 2015. The role of CD95 and CD95 ligand in cancer. Cell Death Differ. 22: 549-559.

26. Fulda S, Debatin KM. 2006. Extrinsic versus intrinsic apoptosis pathways in anticancer chemotherapy. Oncogene 25: 4798-4811.

27. Kim Y, Cha S, Seo T. 2016. Activation of the phosphatidylinositol 3-kinase/Akt pathway by viral interferon regulatory factor 2 of Kaposi's sarcoma-associated herpesvirus. Biochem. Biophys. Res. Commun. 470: 650-656.

28. Choi YB, Sandford G, Nicholas J. 2012. Human herpesvirus 8 interferon regulatory factor-mediated $\mathrm{BH} 3$-only protein inhibition via Bid BH3-B mimicry. PLoS Pathog. 8: e1002748.

29. Hwang KY, Choi YB. 2016. Modulation of mitochondrial antiviral signaling by human herpesvirus 8 interferon regulatory factor 1. J. Virol. 90: 506-520. 
30. Vogelstein B, Lane D, Levine AJ. 2000. Surfing the p53 network. Nature 408: 307-310.

31. Lee HR, Kim MH, Lee JS, Liang C, Jung JU. 2009. Viral interferon regulatory factors. J. Interferon Cytokine Res. 29: 621-627.

32. Wang S, Zhao Y, Aguilar A, Bernard D, Yang CY. 2017. Targeting the MDM2-p53 Protein-Protein Interaction for New Cancer Therapy: Progress and Challenges. Cold Spring Harb Perspect Med. 7(5). pii: a026245..

33. Vazquez A, Bond EE, Levine AJ, Bond GL. 2008. The genetics of the p53 pathway, apoptosis and cancer therapy. Nat. Rev. Drug Discov. 7: 979-987.

34. Shin YC, Nakamura H, Liang $X$, Feng P, Chang H, Kowalik $\mathrm{TF}$, et al. 2006. Inhibition of the ATM/p53 signal transduction pathway by Kaposi's sarcoma-associated herpesvirus interferon regulatory factor 1. J. Virol. 80: 2257-2266.

35. Seo T, Park J, Lee D, Hwang SG, Choe J. 2001.Viral interferon regulatory factor 1 of Kaposi's sarcoma-associated herpesvirus binds to $\mathrm{p} 53$ and represses p53-dependent transcription and apoptosis. J. Virol. 75: 6193-6198.

36. Chavoshi S, Egorova O, Lacdao IK, Farhadi S, Sheng Y, Saridakis V. 2016. Identification of Kaposi Sarcoma Herpesvirus (KSHV) vIRF1 protein as a novel interaction partner of human Deubiquitinase USP7. J. Biol. Chem. 291: 6281-6291.

37. Lee HR, Toth Z, Shin YC, Lee JS, Chang H, Gu W, et al. 2009. Kaposi's sarcoma-associated herpesvirus viral interferon regulatory factor 4 targets MDM2 to deregulate the p53 tumor suppressor pathway. J. Virol. 83: 6739-6747.

38. Lee HR, Choi WC, Lee S, Hwang J, Hwang E, Guchhait K, et al. 2011. Bilateral inhibition of HAUSP deubiquitinase by a viral interferon regulatory factor protein. Nat. Struct. Mol. Biol. 18: 1336-1344.

39. Pantry SN, Medveczky PG. 2009. Epigenetic regulation of Kaposi's sarcoma-associated herpesvirus replication. Semin. Cancer Biol. 19: 153-157.

40. Baresova P, Pitha PM, Lubyova B.2013. Distinct roles of Kaposi's sarcoma-associated herpesvirus-encoded viral interferon regulatory factors in inflammatory response and cancer. J. Virol. 87: 9398-9410.

41. Takaoka A, Tamura T, Taniguchi T. 2008. Interferon regulatory factor family of transcription factors and regulation of oncogenesis. Cancer Sci. 99: 467-478.

42. Park J, Lee MS, Yoo SM, Jeong KW, Lee D, Choe J, et al. 2007. Identification of the DNA sequence interacting with Kaposi's sarcoma-associated herpesvirus viral interferon regulatory factor 1. J. Virol. 81: 12680-12684.

43. Hew K, Dahlroth SL, Venkatachalam R, Nasertorabi F, Lim BT, Cornvik T, et al. 2013. The crystal structure of the DNAbinding domain of VIRF-1 from the oncogenic KSHV reveals a conserved fold for DNA binding and reinforces its role as a transcription factor. Nucleic Acids Res. 41: 4295-4306.
44. Seo T, Park J, Choe J. 2005. Kaposi's sarcoma-associated herpesvirus viral IFN regulatory factor 1 inhibits transforming growth factor-beta signaling. Cancer Res. 65: 1738-1747.

45. Hu H, Dong J, Liang D, Gao Z, Bai L, Sun R, et al. 2016. Genome-wide mapping of the binding sites and structural analysis of Kaposi's sarcoma-associated herpesvirus viral interferon regulatory factor 2 reveal that it is a DNAbinding transcription factor. J. Virol. 290: 1158-1168.

46. Koch S, Damas M, Freise A, Hage E, Dhingra A, Ruckert J, et al. 20019. Kaposi's sarcoma-associated herpesvirus vIRF2 protein utilizes an IFN-dependent pathway to regulate viral early gene expression. PLoS Pathog. 15: e1007743.

47. Lee HR, Li F, Choi UY, Yu HR, Aldrovandi GM, Feng P, et al. 2018. Deregulation of HDAC5 by viral interferon regulatory factor 3 plays an essential role in Kaposi's sarcomaassociated herpesvirus-induced lymphangiogenesis. Mbio 9(1). pii: e02217-17

48. Rivas C, Thlick AE, Parravicini C, Moore PS, Chang Y. 2001. Kaposi's sarcoma-associated herpesvirus LANA2 is a B-cellspecific latent viral protein that inhibits p53. J. Virol. 75: 429-438.

49. Xi X, Persson LM, O'Brien MW, Mohr I, Wilson AC. 2012. Cooperation between viral interferon regulatory factor 4 and RTA to activate a subset of Kaposi's sarcoma-associated herpesvirus lytic promoters. J. Virol. 86: 1021-1033.

50. Lee HR, Doganay S, Chung B, Toth Z, Brulois K, Lee S, et al. 2014. Kaposi's sarcoma-associated herpesvirus viral interferon regulatory factor 4 (vIRF4) targets expression of cellular IRF4 and the Myc gene to facilitate lytic replication. J. Virol. 88: 2183-2194.

51. Yu HR, Kim YJ, Lee HR. 2018. KSHV vIRF4 enhances BCL6 transcription via downregulation of IRF4 expression. Biochem. Biophys. Res. Commun. 496: 1128-1133.

52. Shaffer AL, Emre NC, Romesser PB, Staudt LM. IRF4: Immunity. Malignancy! Therapy? Clin. Cancer Res. 15: 29542961.

53. Lee HR, Mitra J, Lee S, Gao SJ, Oh TK, Kim MH, et al. 2016. Kaposi's sarcoma-associated herpesvirus viral interferon regulatory factor 4 (vIRF4) perturbs the G1-S cell cycle progression via deregulation of the cyclin D1 gene. J. Virol. 90: 1139-1143.

54. Robinson BA, Estep RD, Messaoudi I, Rogers KS, Wong SW. 2012. Viral interferon regulatory factors decrease the induction of type I and type II interferon during rhesus macaque rhadinovirus infection. J. Virol. 86: 2197-2211.

55. Springgay LK, Fitzpatrick K, Park B, Estep RD, Wong SW. 2019. Rhesus macaque rhadinovirus encodes a viral interferon regulatory factor to disrupt promyelocytic leukemia nuclear bodies and antagonize type I interferon signaling. J. Virol. 93(6). pii: e02147-18. 